\title{
Tolerability of antiretroviral therapy (HAART) in elderly age
}

\author{
B M Celesia \\ From de Senectute: Age and Health Forum \\ Catanzaro, Italy. 5-7 December 2009
}

\section{Background}

The median age of people living with HIV/AIDS is increasing: while efficacy of HAART has reduced mortality and prolonged the life of patients, low perception of risk and new late diagnosis are related to infection in an elderly age. Most new late diagnosis are performed in older hardly immuno-compromised subjects; the development of opportunistic diseases complicates management of therapeutic strategies (drugs interactions, cumulative toxicities, hypersensitivity reaction), mainly in presence of comorbities.

Most randomized clinical trials with new drugs tend to avoid the enrolment of $>50$ years patients with proper comorbities of old age. So the few data we can analyze come from observational cohorts.

While it is well defined that older people are more adherent to treatment and achieved an adequate control of HIV-RNA viremia more frequently than younger although an incomplete immuno-reconstitution, the development of drug toxicity with higher frequency is controversial.

Elderly people are more frequent to show reduced hepatic and kidney function, frequently have comorbities requesting new therapies with a higher pill burden and higher risk of drug interactions. Most antiretroviral drugs are metabolized by cytochrome system. Treatment may accelerate mitochondrial dysfunction, nucleoside reverse transcriptase inhibitors may contribute to muscle wasting. Some typical side effects of HAART such as body change and facial lypoatrophy could be more evident when associated with natural facial changes occurring with aging and modify negatively self perceived body image and relationship capacity. Age was also shown to increase the risk of renal tubular disease in association with tenofovir.

\footnotetext{
Unit of Infectious diseases University of Catania ARNAS Garibaldi, Catania, Italy
}

Age is one of the major cardio-vascular disease (CVD) risk factors and the inflammatory component may contribute to this increased risk despite HAART efficacy. The risk of myocardial infarctions is increased in older treated HIV+ patients: metabolic abnormalities are more frequent at baseline and the treatment could increase the cumulative risk of CVD.

\section{Conclusions}

Tolerability of HAART seems do not differ in older vs younger patients although new cardiovascular, endocrine, metabolic or neurological events are more probable in the first group.

An increasing number of people older than 50 years old will attend our clinics in the near future. It'll be necessary to monitor short and long term toxicity and consider the different pharmacokinetics of drugs in an older body to perform adequate therapeutic strategies and to reduce risk of drugs interactions.

The care of patients with HIV will probably become more complex and more specialists will called on to help manage this complex disease.

Published: 19 May 2010

doi:10.1186/1471-2318-10-S1-L34

Cite this article as: Celesia: Tolerability of antiretroviral therapy (HAART)

in elderly age. BMC Geriatrics 2010 10(Suppl 1):L34. 